\title{
HSPA12B Expression as an Epigenetic Signature Following Ischemic Stroke: Heat Shock Protein Mini Review
}

\author{
Hunter Rolain, Justyn Nguyen, Gurwattan S Miranpuri, Youn Youngwon, Reid Alisch and \\ Azam S Ahmed* \\ Department of Neurological Surgery, University of Wisconsin School of Medicine and Public Health, USA
}

*Corresponding author: Azam S Ahmed, MD, Department of Neurological Surgery, University of Wisconsin School of Medicine Public Health. Madison, WI 53792, USA.

To Cite This Article: Hunter Rolain, Justyn Nguyen, Gurwattan S Miranpuri, Youn Youngwon, Reid Alisch, Azam S Ahmed, HSPA12B Expression as an Epigenetic Signature Following Ischemic Stroke: Heat Shock Protein Mini Review. Am J Biomed Sci \& Res. 2021 - 11(4). AJBSR.MS.ID.001644.

DOI: 10.34297/AJBSR.2021.11.001644.

Received: 䟧 December 03, 2020; Published: 㘹 January 08, 2021

\begin{abstract}
Stroke is a cardiovascular condition that caused by a clot or obstruction that prevents blood from flowing to the brain. Neural cells within the occluded area undergo cell death within minutes, and the results are often permanent, making stroke one of the leading causes of serious longterm disability. Stroke is a complex disorder that requires innovative solutions to attenuate the effects of stroke and improve clinical outcomes. Traditionally, stroke treated by relieving vascular occlusions and restoring blood flow to the infarcted tissue (necrotic tissue; core infarct) and the surrounding tissue at risk (penumbra). This technique known as thrombolysis, utilizing both chemical and mechanical modes. Chemical thrombolysis utilizes a catheter that administers medication to the clot site, which includes a multitude of treatments that include intravenous (IV) thrombolysis with recombinant tissue plasminogen activator, or rTPA, and intra-arterial (IA) therapies that aim to directly remove the occlusive clot and reestablish blood flow through recanalization.

This recanalization is ultimately, what allows the ischemic tissue to be repaired. By only focusing on revascularization, we miss a unique opportunity to study pathophysiology and biomarkers within the penumbric brain, which are key to understanding stroke [1,2]. The understanding of this pathophysiology will lead to improved clinical outcomes of acute ischemic stroke (AIS) patients. Heat shock proteins (Hsp), a well-characterized class of proteins, have been implicated in this relative sensitivity and are known to be rapidly inducible through heat shock factors (HSF) binding to heat shock elements (HSE). The expression of the novel heat shock protein HSPA12B, belonging to the HSP 70 family, has been recently uncovered as an imperative epigenetic process that modulates physiological conditions post-ischemia. This mini review recapitulates some of the most promising data that gives support to HSPA12B's clinical relevance in ischemic patients.
\end{abstract}

\section{Introduction}

Stroke is a cardiovascular condition that caused by a clot or obstruction that prevents blood from flowing to the brain. Neural cells within the occluded area undergo cell death within minutes, and the results are often permanent, making stroke one of the leading causes of serious long-term disability. Stroke is a complex disorder that requires innovative solutions to attenuate the effects of stroke and improve clinical outcomes. Traditionally, stroke treated by relieving vascular occlusions and restoring blood flow to the infarcted tissue (necrotic tissue; core infarct) and the surrounding tissue at risk (penumbra). This technique known as thrombolysis, utilizing both chemical and mechanical modes. Chemical thrombolysis utilizes a catheter that administers medication to the clot site, which includes a multitude of treatments that include intravenous (IV) thrombolysis with recombinant tissue plasminogen activator, or rTPA, and intra-arterial (IA) therapies that aim to directly remove the occlusive clot and reestablish blood 
flow through recanalization. This recanalization is ultimately, what allows the ischemic tissue to be repaired.

By only focusing on revascularization, we miss a unique opportunity to study pathophysiology and biomarkers within the penumbric brain, which are key to understanding stroke [1,2]. The understanding of this pathophysiology will lead to improved clinical outcomes of acute ischemic stroke (AIS) patients. Heat shock proteins (HSPs), a well-characterized class of proteins, have been implicated in this relative sensitivity and are known to be rapidly inducible through heat shock factors (HSF) binding to heat shock elements (HSE). It is evident by using animal models that HSP70 proteins only synthesized in neurons peripheral to the ischemic core. Furthermore, overexpression of HSP70 protein in transgenic mice markedly protected tissue against infarction [3]. These differences in protein expression and overall ischemia sensitivity may be due to genetic and epigenetic factors. Moreover, research has shown that epigenetic modifications (e.g., DNA methylation) in a variety of processes from angiogenesis to oxidative stress affect neuroplasticity in the stroke recovery period [4]. These include an array of cytosine-guanine (CpG) methylation for DNA silencing [5], chromatin modifications [6], and miRNA post-transcriptional expression modification [7] in a variety of processes from angiogenesis to oxidative stress protection.

\section{Heat Shock Protein, Epigenetic Expression and} Modification

Heat shock proteins (HSPs), existing virtually in all living organisms, are a class of proteins defined by their kilodalton (Kd) weight. HSPs expressed in accordance with exposures to stressful conditions, including heat, UV light, wound healing, tissue remodeling, and other stressors. This regulatory mechanism originally discovered in Drosophila and later ascribed to be the heat shock response. Acting as a chaperone to innumerable biological mechanisms, heat shock proteins aid in protein synthesis by ensuring correcting protein folding and stabilization of newly generated proteins and corollary structures. Described as a genetic mechanism, heat shock proteins are transcriptionally regulated, alluding to the possibility of modulation of heat shock protein transcriptional control mechanisms, known as heat shock factors (HSF). By modulating expression of HSP through transcriptional regulation, the heat shock response can be induced/diminished to achieve novel clinical outcomes; possibly enhancing and bolstering intrinsic biological repair pathways to assist functional recovery following injury, particularly in stroke [8].

\section{HSPA12B}

The mammalian heat shock protein A12B (HSPA12B) is native to the 70-Kd HSP family. Unlike other HSP70's that are considered to be ubiquitously expressed, HSPA12B has been found to be mainly expressed in vascular endothelial tissue of the brain, heart, kidney, and lungs through which its relevance is ascribed to several biological functions. Most notably, HSPA12B significantly contributes to organ growth and repair, recovery of vascular tissue, and chronic conditions such as ischemia. As described in recent studies and further affirmed through translational animal model testing, HSPA12B has been ascribed to contribute to imperative biological functions through maintenance of angiogenic activities, modulating cellular adhesion and cell migration, and other modes of function. Therefore, the HSPA12B protein is a highly peculiar and specific member of the HSP70 family. However, being relatively novel, the HSPA12B protein as a mechanistic target for abating chronic diseases may be profound. However, particularly within neural tissue, which researchers have experimentally determined high levels of HSPA12B expression, modulation of HSPA12B as a biomarker for neural ischemia may be indicative of its pertinence to ischemic stroke and associated conditions [8]

\section{HSPA12B Angiogenesis}

Reperfusion due to angiogenesis is crucial for the recovery of the ischemic area of your brain after stroke [8]. Angiogenesis helps promote neurogenesis and neuronal plasticity, allowing for greater functional recovery of the brain after ischemic conditions. In recent research, the HSP70 isoform, HSPA12B, has been linked to this stroke pathophysiology due to its active role in endothelial cells and the early stages of angiogenesis, which are crucial for neuronal recovery. In a study of zebrafish blood vessels, RNAi of fluorescently tagged HSPA12B led to stunted in vitro angiogenesis due to decreased endothelial cell adhesion as well as the limited migration of human umbilical vein endothelial cells (HUVECs) to the site of injury [9]. One possible mechanism discussed in recent research in vivo rodent testing for the role of HSPA12B in angiogenesis is that the protein expression of HSPA12B inhibits anti-angiogenic proteins such as AKAP12 $[9,10]$. Specifically, knockdown HSPA12B mutants led to increased AKAP12 protein levels, which ultimately inhibits vascular endothelial growth factor (VEGF) crucial for angiogenesis by $75 \%$ [9]. While the involvement of HSPA12B in stroke not entirely understood due to its involvement in numerous biological pathways, HSPA12B's involvement in angiogenesis is a promising epigenetic treatment for ischemic stroke.

\section{MicroRNAs in Transcriptional Regulation of HSPA12B}

MicroRNAs (miRNAs) are a class of small, non-coding RNA that are involved in the regulation of gene expression at the posttranscriptional level by degrading specific mRNAs. In recent research of oxygen-glucose deprivation (OGD) N2A in vitro cells and in vivo MCAO-induced ischemic stroke mouse brain cells, the controlled expression of miRNAs has provided promise in regulating neurological diseases and have led to improved neurological outcomes through the regulation of HSPA12B [11]. One of the miRNAs that has shown implications in stroke and myocardial 
infarction pathophysiology is miR-134. MiR-134 is suggested to target the 3' UTR of HSPA12B and inhibit HSPA12B protein expression. From the research, it was found that overexpression of miR-134, in 1-hour OGD N2A cells or 1-hour MCAO brain cells, exacerbated cell death and apoptosis by downregulating the expression of HSP12AB 12 hours to 7 days after reperfusion. On the other hand, downregulation of miR-134 reduced cell death and apoptosis due to increased HSP12AB levels. This leads to improved neurological outcomes by inhibiting infarct growth.

\section{eNOS dependent mechanism section}

Upon further determination of the HSPA12B's relevance in functional recovery for ischemic conditions, researchers determined that the endothelial cells situated at the interface between the vessel wall and the blood barrier demonstrated an imperative role in modulating vascular tone and homeostasis, specifically in determining pro and anti-atherosclerotic gene expression. In the form of fluid blood shear stress and cyclic strain on the vascular wall, hemodynamic stimuli on the luminal face of endothelial cells resulted in the release of the vasodilator nitric oxide. Regulated through the endothelial nitric oxide synthase response (eNOS), the production of nitric oxide synthase proteins aid in the production of nitric oxide. While attenuation of the eNOS response has been found to result in several patho-physiological disorders, purposeful modulation of the eNOS response can be a viable mode for functional recovery, particularly in ischemic stroke [12] (Figure 1).

The inhibition of the eNOS response may induce favorable outcomes in ischemic conditions [13]. In transgenic mice with overexpressed HSPA12B protein and wild-type mice, middle cerebral artery occlusion induced for 60 minutes, followed by reperfusion at various time points. To develop understanding for HSPA12B in functional recovery, HSPA12B expression measured using an immunoblotting analysis. Found in the immunoblotting assay, [13] demonstrated that HSPA12B continually overexpressed for seven days following ischemic stroke in both the transgenic and wild type mice. However, in corroboration with the conditional hypothesis of HSPA12B expression, functional recovery measures assessed. While functional recovery examined in both the transgenic and wild type mice [13]. discovered that HSPA12B overexpression in trangenic mice resulted in statistically significant recovery in the functional recovery tests. Therefore, HSPA12B transgeneic mice demonstrated improved survival rate, neurological and locomotor activity post-ischemia. In conjuction with functional recovery tests and protein synthesis assays, [13]. determined that the HSPA12B response, and associated functional recovery, heavily regulated by the production of nitric oxide synthases following ischemic conditions. At the infarct site for ischemic wild type and overexpressed HSPA12B mice, immunoblotting assays determined that the expression of nitric oxide synthases were significantly upregulated in comparison to the SHAM control mice. In conjunction with HSPA12B expression assays and functional recovery tests [13] discovered in HSPA12B overexpressed mice that administration of the eNOS inhibitor L-NAME diminished functional recovery and survival rate post ischemia than in overexpressed HSPA12B mice that did not receive the eNOS inhibitor L-NAME. Therefore, the derivation of the findings suggests that while HSPA12B overexpression increases survivability and functional recovery post-ischemia, the attenuation of functional recovery and survivability following the administration of the eNOS inhibitor L-NAME demonstrates that the eNOS response is a regulatory mechanism in the expression of HSPA12B post-ischemia.

\section{PI3K/Akt-Dependent Reperfusion}

In recent research of transgenic mice overexpressing human HSPA12B, cardiac protection controlled by the activation of the phosphoinositide-3-kinase/protein kinase B (PI3K/Akt) signaling pathway. With similarities in myocardial ischemia and brain ischemia, it was suggested to observe this pathway in the brain as well. After mice experienced 1 hour of MCAO, Tg HSPA12B mice showed decreased infarct volumes and improved neurological outcomes due to increased blood brain barrier integrity and increased endothelial cell migration to the site of injury [14]. Thus, the epigenetic control of HSPA12B shows a promising therapeutic benefit for acute ischemic stroke.

\section{Future Perspective}

By designing and employing individualized approaches to healthcare, novel therapeutic approaches aim to treat the patient rather than just the disease. As such, it is paramount that future studies delve into the complex mechanisms of genetics and bio molecular interactions from an epigenetic standpoint. Interventions and research on the treatments of stroke have largely focused on relieving vascular occlusions and restoring blood flow to the infarcted tissue (necrotic tissue; core infarct) and the surrounding tissue at risk (penumbra). These include a multitude of treatments that include intravenous (IV) thrombolysis with recombinant tissue plasminogen activator, or rTPA, and intra-arterial (IA) therapies that aim to directly remove the occlusive clot and reestablish blood flow (recanalization). IV rTPA effectiveness is highly time dependent and therefore only administered within 4.5 hours of symptom onset. Its effectiveness directly related to the "clot burden" with longer thrombus in larger vessels less likely to recanalize. Initially, IA thrombectomy reserved for patients presenting within 6 hours of "last known well".

However, recently this time window has expanded to 24 hours primarily due to improved patient selection using radiographic (CT or MR) perfusion parameters to assess the volume of infarcted (i.e., irreversibly injured) versus ischemic (potentially reversible) brain. Studies also have shown that patients have differing susceptibilities 
and resistances to infarction growth, based on collateral circulation pathways and other biologic differences inherent to individual genetic, epigenetic, and proteomic factors. Heat shock proteins, a well-characterized class of proteins, have been implicated in this relative sensitivity and are known to be rapidly inducible through heat shock factors (HSF) binding to heat shock elements (HSE).

In particular, the 70 kilodalton (hsp70) family has been implicated in stroke and results in both induction and arrest of inflammation at different time points during ischemic stroke. Stress protein release occurs both through physiological secretion mechanisms and during cell death by necrosis. Hsp60 and hsp70 are able to enter the bloodstream and may possess the ability to act at distant sites in the body. Many of the effects of extracellular stress proteins Hsp 60 and Hsp70 mediated through cell surface receptors. Such receptors include Toll like Receptors 2 and 4, CD40, CD91, CCR5 and members of the scavenger receptor family such as LOX-1 and SREC-1. Specific subtypes of cardiovascular disease (CVD) where HSP60 implicated in the initiation and/or progression of disease [16] (Figure 2). Figure 2 Suggested developed timeline for thrombectomy and imaging study beginning with the onset of stroke, performing thrombectomy, and imaging at necessary time intervals (Dr. Azam S Ahmed, Stroke laboratory, Neurological Surgery, UW Madison, WI). Following the onset of stroke, patients screened for eligibility. Following screening, consent is given, and blood samples taken for HSP60/70 detection assay.

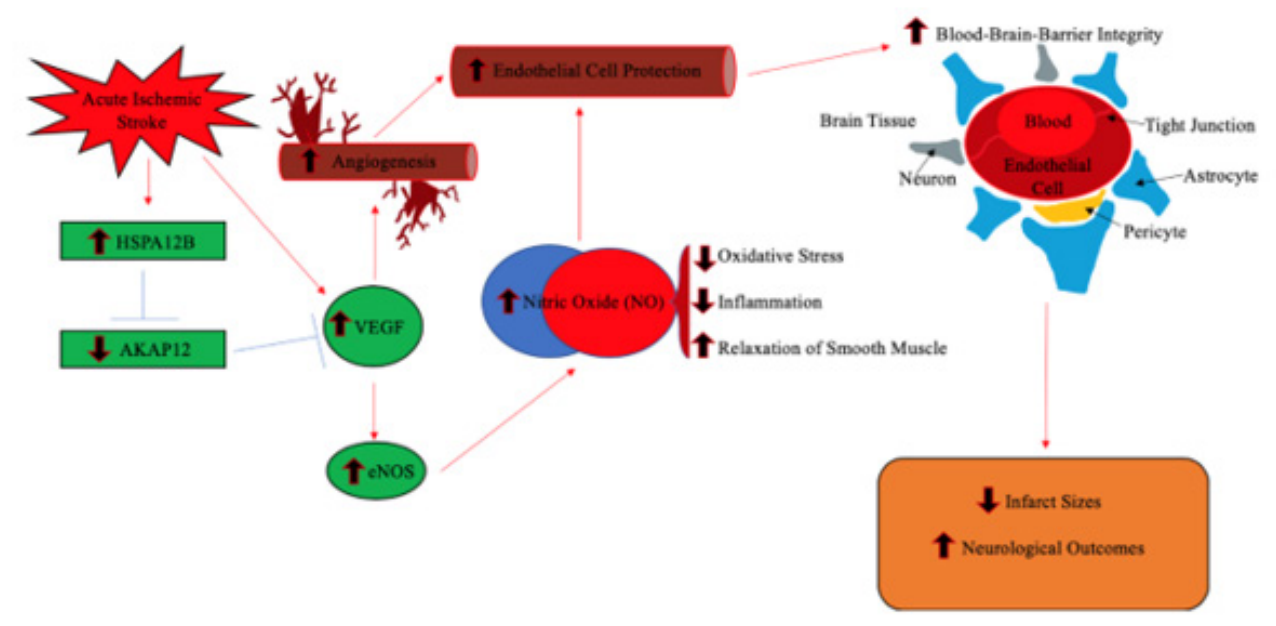

Figure 1: In response to stress, the eNOS response regulated the production of the protein nitric oxide synthase. Nitric oxide synthases are a class of proteins that produce the vasodilator nitric oxide. In conjunction with the expression of the HSPA12B protein, the eNOS response has been a targeted mechanism for increasing favorability of survival and recovery post ischemia.

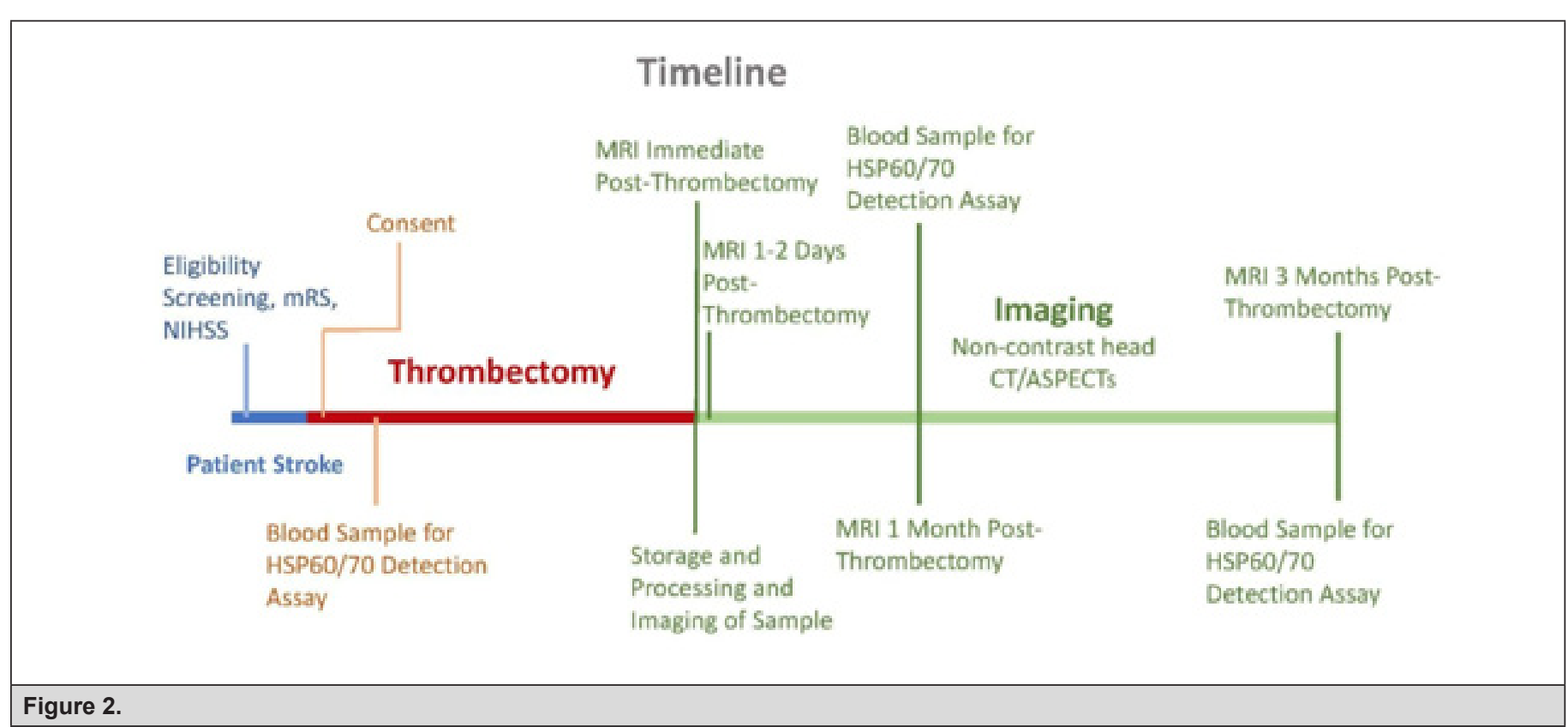


Thrombectomy performed and followed by three months of imaging and blood sampling for continued HSP60/70 detection assay. Moreover, research has shown that epigenetic modifications (e.g., DNA methylation) in a variety of processes from angiogenesis to oxidative stress affect neuroplasticity in the stroke recovery period. In our research direction, we hypothesize that DNA methylation abundance across the human genome dictates patient susceptibility and resistance to infarction in the penumbra. Here we propose to define the unique DNA methylation signatures of penumbric brain and peripheral blood following stroke and develop a blood-based biomarker panel for stroke diagnosis, to improve estimates of stroke prognosis, and to guide personalized treatment of stroke. Importantly, proposed analyses will include clinical observations and state-of-the-art radiographic imaging as continuous variables. Determining the molecular physiology of the penumbric tissue creates an entirely new avenue for potential therapies targeted toward neuroprotection. Ultimately, these molecular signatures can be used to elucidate neuroprotective factors and identify screening markers to identify patients at high risk for increased infarction growth.

\section{References}

1. Yiwang Guo Y, Li P, Guo Q Shang K, Yan D, et al. (2013) Pathophysiology and Biomarkers in Acute Ischemic Stroke - A Review. Tropical Journal of Pharmaceutical Research 12 (6): 1097-1105.

2. Allard L, Burkhard PR, Lescuyer P, Burgess JA, Walter N, et al. (2005) PARK7 and nucleoside diphosphate kinase A as plasma markers for the early diagnosis of stroke. Clin chem 51(11): 2043-2051.

3. Zhan X, Kim C, Sharp FR (2008) Very brief focal ischemia simulating transient ischemic attacks (TIAs) can injure brain and induce Hsp70 protein. Brain Res 1234: 183-197.

4. Felling RJ, Song H (2015) Epigenetic mechanisms of neuroplasticity and the implications for stroke recovery. Exp Neurol 268: 37-45.
5. Endres M, Meisel A, Biniskiewicz D, Namura S, Prass K, et al. (2000) DNA methytransferase contributes to delayed ischemia brain injury. J Neurosci 20(9): 3175-3181.

6. Elder J, Cortes M, Rykman A, Hill J, Karuppagounder S, et al. (2013) The epigenetics of stroke recovery and rehabilitation: from polycomb to histone deacetylases. Neurotherapeutics 10(4): 808-816.

7. Ouyang L, Shi Z, Zhao S, Wang FT, Zhou TT, et al. (2012) Programmed cell death pathways in cancer: a review of apoptosis, autophagy and programmed necrosis. Cell Prolif 45(6): 487-498.

8. Ergul A, Ahmed A, Susan C Fagan (2012) Angiogenesis a harmonized target for recovery after stroke. Stroke 43: 2270-2274.

9. Rebecca J Steagall , Antonio E Rusiñol, Quynh A Truong, Zhihua Han (2006) HSPA12B is predominantly expressed in endothelial cells and required for angiogenesis. Arterioscler Thromb Vasc Biol 26(9): 20122018.

10. Fouad A Zouein, Mazen Kurdi, George W Booz (2013) HSPA12B and repairing the heart: beauty in simplicity. Cardiovasc Res 99(4): 587-589.

11. Chi W, Meng F, Li Y, Wang Q, Wang G, et al. (2014) Downregulation of miRNA-134 protects neural cells against ischemic injury in N2A cells and mouse brain with ischemic stroke by targeting HSPA12B. Neuroscience 277: $111-122$

12. Fleming, Ingrid (2010) Molecular mechanisms underlying the activation of eNOS. Pflügers Arch 459(6): 793-806.

13. Zhao Y, Liu C, Liu J, Kong Q Mao Y, et al. (2018) HSPA12B promotes functional recovery after ischemic stroke through an eNOS-dependent mechanism. J Cell Mol Med 22(4): 2252-2262.

14. Yujie Ma, Chen Lu, Chuanfu Li, Rongrong Li, Yangyang Zhang, et al. (2013) Overexpression of HSPA12B protects against cerebral ischemia/ reperfusion injury via a PI3K/Akt-dependent mechanism. Biochim Biophys Acta 1832(1): 57-66.

15. Calderwood SK, Mambula SS, Gray Jr, Jimmy R Theriault (2007) Theriault JR. Extracellular heat shock proteins in cell signaling. FEBS Lett 581(19): 3689-3694.

16. Manfredi Rizzo, Alberto JL Macario, Everly Conway de Macario, Ioanna Gouni-Berthold, Heiner K Berthold, et al. (2011) Heat Shock Protein-60 and Risk for Cardiovascular Disease. Current Pharmaceutical Design17(33): 3662-3668. 